\title{
Recent STAR Results from Heavy-Ion and Polarized Proton Programs
}

\author{
Grigory Nigmatkulov ${ }^{1, *}$ for the STAR Collaboration \\ ${ }^{1}$ National Research Nuclear University MEPhI \\ 115409, Kashirskoe shosse 31, Moscow, Russia
}

\begin{abstract}
We present recent physics results from the STAR experiment at Relativistic Heavy Ion Collider (RHIC). The proceedings cover studies of azimuthal anisotropy in small and large systems, global and local hyperon polarization, correlation femtoscopy, antideuteron and $J / \psi$ production from heavyion program as well as the measurements of longitudinal spin asymmetry from polarized proton program.
\end{abstract}

\section{Introduction}

The main goal of high-energy physics is to understand the properties of the strong interaction that can be described by the Quantum Chromodynamics (QCD). In order to measure the QCD matter, one can use ion-ion collisions. Variation of collision energy provides a unique opportunity to investigate the properties of the created medium on baryon chemical potential $\left(\mu_{B}\right)$ and temperature $(T)$. This medium is also known as Quark-Gluon Plasma (QGP). The Relativistic Heavy Ion Collider (RHIC) accelerates and collides nuclei from protons to uranium. Using different colliding species gives important information about the influence of the initial conditions, dynamical evolution and transport properties. RHIC is also the only accelarator of polarized proton beams. Studying the high-energy polorized $p+p$ collisions is essential key in understanding of the spin structure of the proton in terms of gluon, quark and antiquark constitiuents. In these proceedings, we report recent results from heavy-ion and polorized proton-proton programs obtained in the Solenoidal Tracker At RHIC (STAR) experiment.

\section{Azimuthal harmonics in small and large systems}

The process of particle emission in the transverse plane is anisotropic. This anisotropy, known as anisotropic flow, can be measured via Fourier decomposition of the single-particle azimuthal angle $(\phi)$ with respect to the $n^{\text {th }}$-order event plane $\left(\Psi_{n}\right)$ [1]. The first three extracted flow coefficients $v_{1}, v_{2}$, and $v_{3}$ are called directed, elliptic, and triangular flow, respectively. The fluctuations-driven component of $v_{1}$, named $v_{1}^{\text {fluc }}$ is proportional to the dipole asymmetry of the collision system [2,3]. Azimuthal anisotropy is one the key observables because it carries information about the viscous hydrodynamic response to the initial spatial distribution in energy density due to fluctuations and geometry.

\footnotetext{
*e-mail: nigmatkulov@gmail.com;ganigmatkulov@mephi.ru
} 
Figure 1 shows the $\left\langle N_{c h}>\right.$ dependence of the $v_{1}^{\text {fluc }}$ (a), $v_{3}$ (b), and $v_{2}$ (c) flow coefficients [4]. The measurements have been performed for $\mathrm{p}+\mathrm{Au}, \mathrm{d}+\mathrm{Au}, \mathrm{Cu}+\mathrm{Cu}, \mathrm{Cu}+\mathrm{Au}$, and $\mathrm{Au}+\mathrm{Au}$ collision at $\sqrt{s_{N N}}=200 \mathrm{GeV}$ and $\mathrm{U}+\mathrm{U}$ collisions at $s q r t s_{N N}=193 \mathrm{GeV}$. The inset in Fig. 1(a) compares the extracted values of $K \equiv 1 /\left(<N_{c h}><p_{T}^{2}>\right)$, that takes into account the long-range non-flow correlations induced by global momentum conservation, for each system.

For $<N_{c h}>\geq 170$, the $v_{n}$ values show a decrease with increasing values of $<N_{c h}>$. This is consistent with the expected decrease of $\epsilon_{n}$ for more central collision as compared to the midcentral ones. The decrease of $v_{2}$ for $\left\langle N_{c h}\right\rangle \leq 170$ shows the dominant role of size-driven viscous attenuation of the flow harmonics for the give multiplicities.

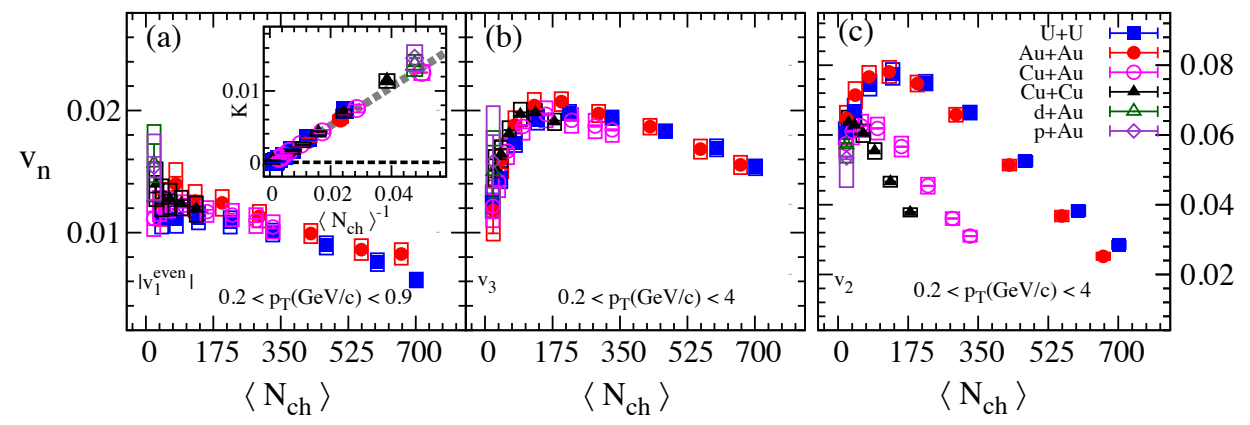

Figure 1. The $v_{1}^{\text {fluc }}$ (a), $v_{3}$ (b), and $v_{2}$ (c) dependence on $<N_{c h}>$ for $\mathrm{p}+\mathrm{Au}, \mathrm{d}+\mathrm{Au}, \mathrm{Cu}+\mathrm{Cu}, \mathrm{Cu}+\mathrm{Au}$, $\mathrm{Au}+\mathrm{Au}$, and $\mathrm{U}+\mathrm{U}$ collision systems. The dashed curve in (c) represents a hydrodynamic model calculation.

The system-dependent behaviour of $v_{2}$ shown in Fig. 1(c) can be attributed to the dependence of $\epsilon_{2}$ on system size for a given value of $\left\langle N_{c h}>\right.$. Figure 2 shows the $v_{2} / \epsilon_{2}$ as a function of $\left\langle N_{c h}\right\rangle^{-1 / 3}$ [4]. This confirms the system dependence of the $\epsilon_{2}$ on system size.

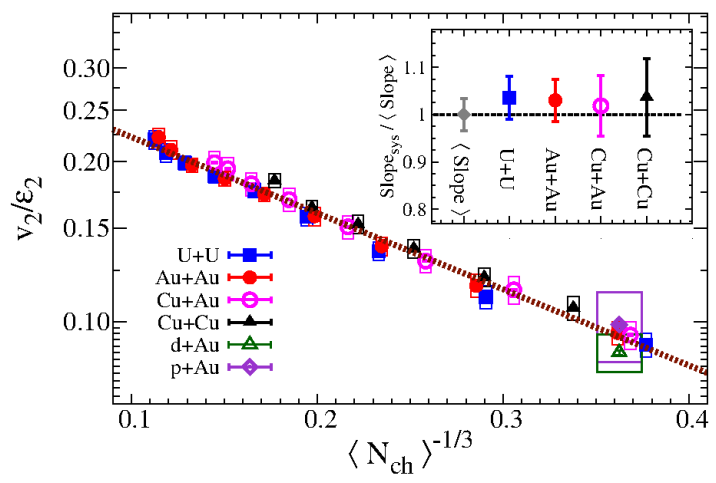

Figure 2. The $v_{2} / \epsilon_{2}$ as a function of $\left\langle N_{c h}\right\rangle^{-1 / 3}$ for $\mathrm{p}+\mathrm{Au}, \mathrm{d}+\mathrm{Au}, \mathrm{Cu}+\mathrm{Cu}, \mathrm{Cu}+\mathrm{Au}, \mathrm{Au}+\mathrm{Au}$, and $\mathrm{U}+\mathrm{U}$ collisions. (Inset) The respective ratios of the slopes extracted for each collision system separately relative to the slope extracted from a fit to the combined data sets. 
The inset in Fig. 2 shows similarity between the slopes of the $\epsilon_{2}$-scaled $v_{2}$ for $\mathrm{Cu}+\mathrm{Cu}$, $\mathrm{Cu}+\mathrm{Au}, \mathrm{Au}+\mathrm{Au}$, and $\mathrm{U}+\mathrm{U}$ collisions over the indicated multiplicity range. The eccentricityscaled results for $\mathrm{p}+\mathrm{Au}$ and $\mathrm{d}+\mathrm{Au}$ collisions also follow the data trend for these heavier collision species with larger systematic uncertainty.

\section{Global hyperon polarization}

The angular momentum carried by colliding nuclei can be transferred to the created system. Due to the spin-orbit coupling, this may lead to the global polarization of particle' spin along the direction of angular momentum of the system [5,6]. Experimentally, such a global polarization can be probed with hyperons via parity-violating weak decays, in which the daughter baryon is preferentially emitted in the direction of the hyperon's spin. In case of antihyperon, the daughter baryon tends to be emitted in the opposite direction to the parent spin.

Since the angular momentum of the system is perpendicular to a plane defined by the impact parameter vector and the beam direction (so-called reaction plane), the hypeon polarization can be measured via the azimuthal distribution of daughter baryons with respect to the reaction plane $\left(\Psi_{1}\right)$ in the hyperon's rest frame $\left(\phi_{p}^{*}\right)$ :

$$
P_{H}=\frac{8}{\pi \alpha_{H}} \frac{<\sin \left(\Psi_{1}-\phi_{p}^{*}\right)>}{\operatorname{Res}\left(\Psi_{1}\right)},
$$

where $\alpha_{H}$ is the hyperon decay constant, $\operatorname{Res}\left(\Psi_{1}\right)$ is the experimental resolution of the firstorder event plane.

Figure 3 shows the global polarization of $\Lambda$ and $\bar{\Lambda}$ hyperons as a function of collision energy $\left(\sqrt{s_{N N}}\right)$ for $20-50 \%$ most central $\mathrm{Au}+\mathrm{Au}$ collisions [7, 8]. The global polarization results follow the same trend and seem to decrease with increasing collision energy.

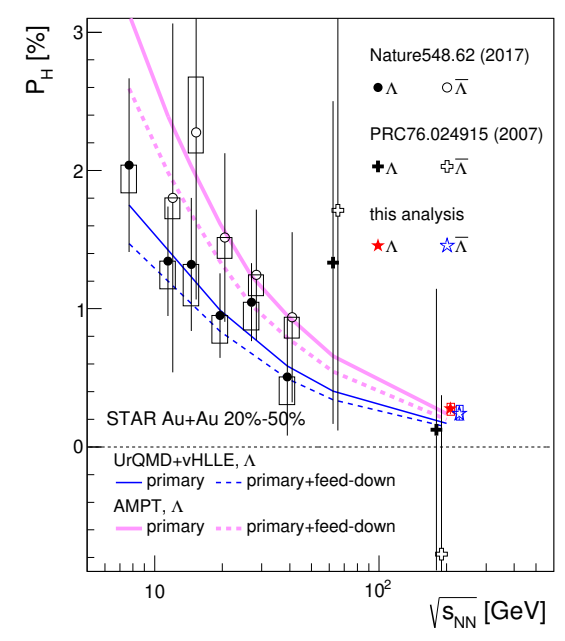

Figure 3. Global polarization of $\Lambda$ and $\bar{\Lambda}$ as a function of collision energy ( $\sqrt{s_{N N}}$ ) for 20-50\% central $\mathrm{Au}+\mathrm{Au}$ collisions. Thin lines show calculations from a $(3+1) \mathrm{D}$ cascade + viscous hydrodynamic model (UrQMD+vHLLE) [9] and bold lines show the AMPT model calculations [10].

The (3+1)D viscous hydrodynamic model vHLLE with the UrQMD initial state [9] agrees with the data over a wide range of collision energies, including $\sqrt{s_{N N}}=200 \mathrm{GeV}$ within 
the current accuracy of the experimental measurements. Calculations from a Multi-Phase Transport (AMPT) model [10] predict slightly higher global polarization than those from the hydrodynamic model, but are also in good agreement with the data within uncertainties.

\section{Polarization along the beam direction}

Recently STAR has also reported the measurement of the hyperon polarization along the beam direction [11]:

$$
P_{z}=\frac{<\cos \left(\theta_{p}^{*}\right)>}{\alpha_{H}<\cos ^{2}\left(\theta_{p}^{*}\right)>} .
$$

The beam direction component of the polarization, $P_{z}$, arises from vorticity due to elliptic flow. It is expected that $P_{z}$ will be more sensitive to the later stages of the system evolution after the anisotropic flow is developed [2] as compared to global hyperon polarization which originates mostly from the initial velocity fields. The $<\cos \left(\theta_{p}^{*}\right)>$ was measured as a function of azimuthal angle of $\Lambda(\bar{\Lambda})$ relative to $\Psi_{2}$. The acceptance effects and inefficiencies were taken into account. Figure 4 shows $<\cos \left(\theta_{p}^{*}\right)>^{s u b}$ of $\Lambda$ and $\bar{\Lambda}$, obtained with event plane method, as a function of azimuthal angle relative to $\Psi_{2}$ for the $20 \%-60 \%$ centrality region.

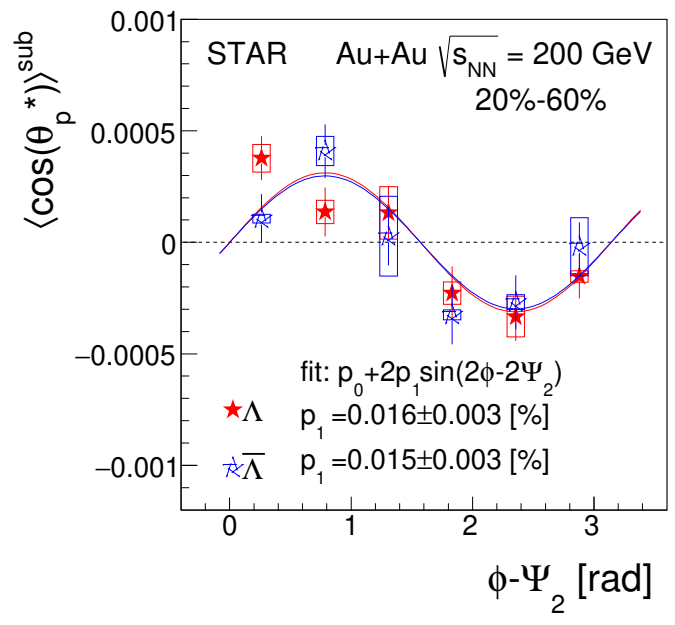

Figure 4. The $<\cos \left(\theta_{p}^{*}\right)>$ of $\Lambda$ and $\bar{\Lambda}$ as a function of azimuthal angle $\phi$ relative to the second-order event plane $\Psi_{2}$ for $20 \%-60 \%$ centrality bin in Au+Au collisions at $\sqrt{s_{N N}}=200 \mathrm{GeV}$. Open boxes show the systematic uncertainties. The lines show the fit with the sine function.

The lines in Fig. 4 represent the fit results using $p_{0}+2 p_{1} \sin \left(2 \phi-2 \Psi_{2}\right)$, where $p_{0}$ and $p_{1}$ are the fit parameters. The data are consistent with a sine structure for both $\bar{\Lambda}$ and $\Lambda$. This is expected due to the elliptic flow.

\section{Antideuteron production}

The underlying mechanism for light (anti)nuclei production in relativistic heavy-ion collisions is not well understood. One of the possible approaches is through coalescence of (anti)nucleons. Since the binding energies of light nuclei are small $(\approx 2.2 \mathrm{MeV}$ for 
(anti)deuteron and $\approx 7.7 \mathrm{MeV}$ for ${ }^{3} \mathrm{He}$ ), the light nuclei cannot survive when the temperature is much higher than the binding energy. The typical kinetic freeze-out temperature for light hadrons is around $100 \mathrm{MeV}$, hence they may be formed by final-state coalescence, after nucleons are decoupled from the hot and dense system. In case of the coalescence picture, the invariant yield of light nuclei is related to the invariant yiled of nucleons as:

$$
E_{a} \frac{d^{3} \mathbf{N}_{A}}{d \mathbf{p}_{A}^{3}} \approx B_{A}\left(E_{p} \frac{d^{3} \mathbf{N}_{p}}{d \mathbf{p}_{p}^{3}}\right)^{A},
$$

where $\mathbf{p}_{p}, \mathbf{p}_{n}$, and $\mathbf{p}_{A}$ are momenta of proton, neutron, and nucleus, respectively. Here $\mathbf{p}_{A}=$ $A \mathbf{p}_{p}$, assuming $\mathbf{p}_{p} \approx \mathbf{p}_{n}$. The $\mathrm{A}$ and $\mathrm{Z}$ are the mass and charge number of the nucleus. The $B_{A}$ is a coalescence parameter and it reflects probability of nucleon coalescence.

Figure 5 shows the excitation function of $B_{2}$ at $p_{T} / A=0.65 \mathrm{GeV} / \mathrm{c}$ in $0 \%-10 \%$ most central $\mathrm{Au}+\mathrm{Au}$ collisions [12].

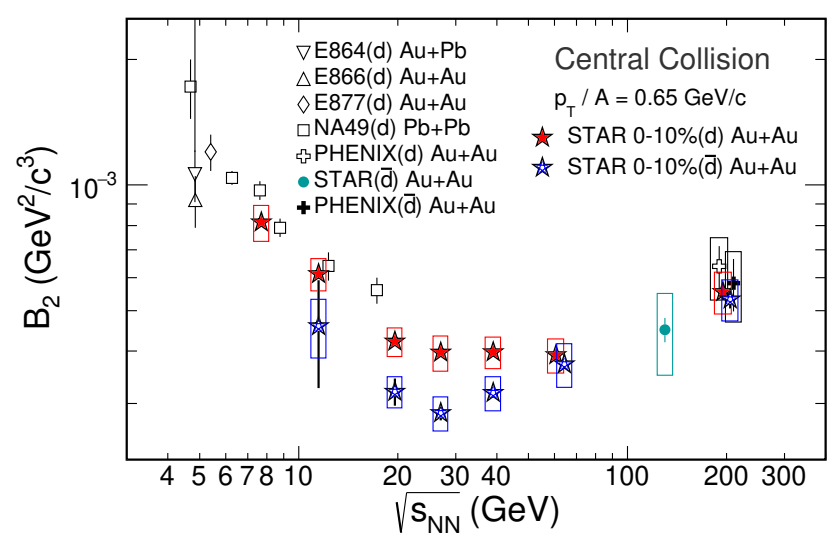

Figure 5. Energy dependence of the coalescence parameter, $B_{2}$, for $d$ and $\bar{d}$ at $p_{T} / A=0.65 \mathrm{GeV} / \mathrm{c}$ from $\mathrm{Au}+\mathrm{Au}$ collisions at RHIC. Results from AGS [13-15], SPS [16-18] (0\%-7\% and 0\%-12\% collision centralities), RHIC $[19,20]\left(0 \%-18 \%\right.$ and $0 \%-20 \%$ collision centrality for $\sqrt{s_{N N}}=130 \mathrm{GeV}$ and $200 \mathrm{GeV}$ ) are also presented.

The results are compared to those measured at AGS [13-15], SPS [16-18] and RHIC [19, 20]. At energies below $\sqrt{s_{N N}}=20 \mathrm{GeV}$, the coalescence parameters $B_{2}$ decrease as a function of increasing collision energy, This implies that the size of the emitting source of nucleons increases with the collision energy. When $\sqrt{s_{N N}}>20 \mathrm{GeV}$, the rate of decrease seems to change and saturate up to $62.4 \mathrm{GeV}$. It might imply a change of the equation of state of the medium in those collisions. The $B_{2}$ from $200 \mathrm{GeV}$ is found to be larger than the BES saturation values, which needs further studies. The $B_{2}$ values for antideuterons are systematically lower than those for deuterons, which implies that the overall size of the antibaryon-emitting source is larger than that of baryons.

\section{Correlation femtoscopy}

In 2015 STAR conducted a fixed-target (FXT) test run using gold ion collisions at $\sqrt{s_{N N}}=4.5 \mathrm{GeV}$ to show that STAR is capable to run in a fixed-target configuration. One beam was circulated in the collider and lowered to directly graze the edge of a $1 \mathrm{~mm}$ thick 
(4\% interaction probability) gold foil target. The target was placed at the edge of the TPC, about $211 \mathrm{~cm}$ away from the center of the detector to make use of the full tracking volume of the TPC. Approximately 1.3 million events were collected with a top $\approx 30 \%$ centrality trigger. Figure 6 (left) shows the measured femtoscopic radii as a function of transverse mass for $0-10 \%$ central $\mathrm{Au}+\mathrm{Au}$ collisions in the fixed-target mode.
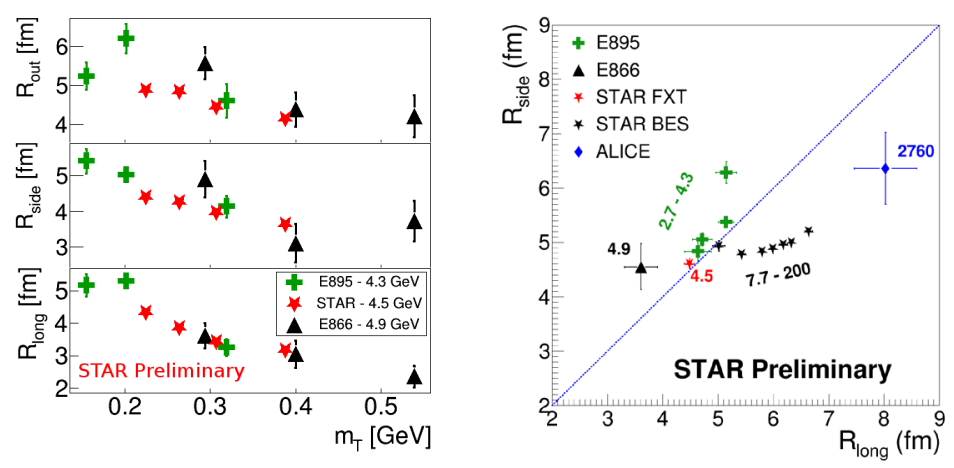

Figure 6. (Left) Transverse mass dependence of $R_{\text {out }}, R_{\text {side }}$, and $R_{\text {long }}$ for pions measured for $0-10 \%$ $\mathrm{Au}+\mathrm{Au}$ collisions in STAR (red stars), E895 (black triangles) [21], and E866 (green crosses) [22]. (Right) The $R_{\text {side }}$ vs. $R_{\text {long }}$ dependence for the E866, E895, STAR [23] and ALICE [24] experiments. Only statistical uncertainties shown.

The STAR FXT results are compared with E895 [21] and E866 (E802) [22] and are consistent with the energy dependence trend of these other experiments within uncertainties. Figure 6 (right) shows the dependence of $R_{\text {side }}$, which reflects the transverse size of the source, on $R_{\text {long }}$ that reflects the size in the longitudinal direction for several collision energies. As the collision energy increases in the FXT regime, compression reduces the source size and increases the baryon density, whereas the BES collider regime shows increasing longitudinal expansion.

The correlation femtoscopy technique was also used to study identical pion correlations in $\mathrm{d}+\mathrm{Au}$ collisions at $\sqrt{s_{N N}}=200 \mathrm{GeV}$. Figure 7 shows the extracted $\pi \pi$ femtoscopic radii, $R_{i n v}$.

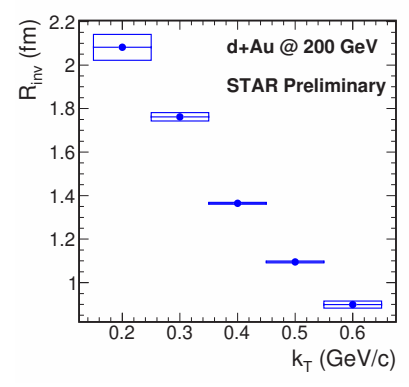

Figure 7. Charged pion femtoscopic as a function of pair transverse momentum, $k_{T}$, measured in $\mathrm{d}+\mathrm{Au}$ collisions at $\sqrt{s_{N N}}=200 \mathrm{GeV}$. Boxes represent systematic uncertainty. 
The $R_{\text {inv }}$ decreases with pair tranverse momentum, $k_{T}=\left|p_{T, 1}+p_{T, 2}\right|$, which is consistent with previuos measurement in $\mathrm{p}+\mathrm{p}$ collisions at the same energy [25].

\section{$7 \mathrm{~J} / \psi$ suppression in $\mathrm{Au}+\mathrm{Au}$ collisions}

Among the various probes of the QGP, quarkonia play a special role as they are expected to dissociate in the medium when the Debye radius, inversely proportional to the medium temperature, becomes smaller than their size. Strong suppression of the $J / \psi$ meson with respect to its yield in $\mathrm{p}+\mathrm{p}$ collisions scaled by the number of binary nucleon-nucleon collisions has been observed at high transverse momenta $\left(p_{T}\right)$ in central heavy-ion collisions at both RHIC and LHC energies. Recently, STAR has presented new measurement of $\mathrm{J} / \psi$ suppression at midrapidity in $\mathrm{Au}+\mathrm{Au}$ collisions at $\sqrt{s_{N N}}=200 \mathrm{GeV}$ through the dimuon decay channel [26] shown for $p_{T}>0.15 \mathrm{GeV} / \mathrm{c}$ in Fig. 8 .

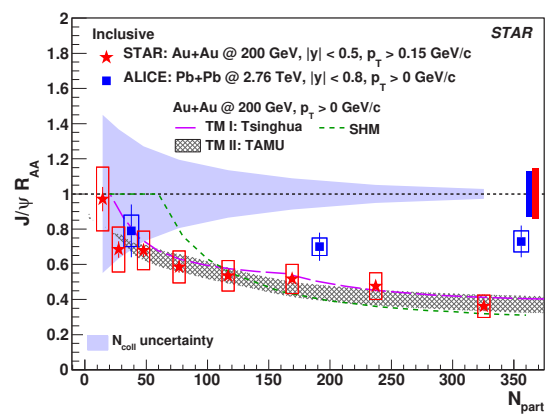

Figure 8. The $\mathrm{J} / \psi R_{A A}$ as a function of $N_{\text {part }}$ above $0.15 \mathrm{GeV} / \mathrm{c}$ in $\mathrm{Au}+\mathrm{Au}$ collisions at $\sqrt{s_{N N}}=200 \mathrm{GeV}$, compared to those for $\mathrm{Pb}+\mathrm{Pb}$ collisions at $\sqrt{s_{N N}}=2.76 \mathrm{TeV}$ [27].

The $\mathrm{J} / \psi R_{A A}$ is seen to decrease from peripheral to central collisions, which is expected in the presence of the QGP. The results are compared to those obtained at $\sqrt{s_{N N}}=2.76 \mathrm{TeV}$ [27]. The low- $p_{T} \mathrm{~J} / \psi$ 's are more suppressed in central and midcentral collisions at RHIC than at the LHC, likely due to the smaller charm quark production cross section and thus smaller regeneration contribution at RHIC. Transport model calculations are consistent with the data at low $p_{T}$. The calculation from Statistical Hadronization Model (SHM) is shown as the dashed line and also describes the data well in non-peripheral events [28].

\section{$8 W^{ \pm}$longitudinal spin asymmetry}

The leptonic $W^{+} \rightarrow e^{+} v$ and $W^{-} \rightarrow e^{-} v$ decay channels provide sensitivity to the helicity distributions of the quarks, $\Delta u$ and $\Delta d$, and antiquarks, $\Delta \bar{u}$ and $\Delta \bar{d}$, that is free of uncertainties associated with nonperturbative fragmentation. The primary observable is the longitudinal single-spin asymmetry $A_{L}=\left(\sigma_{+}-\sigma_{-}\right)=\left(\sigma_{+}+\sigma_{-}\right)$, where $\sigma_{+(-)}$is the cross section when the helicity of the polarized proton beam is positive (negative).

STAR reported new measurements of the single-spin asymmetries for decay positrons and electrons from $W^{ \pm}$bosons produced in longitudinally polarized proton-proton collisions at $\sqrt{s}=510 \mathrm{GeV}$ [29]. The recorded data correspond to an integrated luminosity of about $250 \mathrm{pb}^{-1}$. The luminosity-weighted beam polarization was $P=0.56$, with a relative scale 
uncertainty of $3.3 \%$ for the single-beam polarization and $6.4 \%$ for the product of the polarizations from both beams. The new $W^{ \pm} A_{L}$ data are shown in Fig. 9 and consistent with the previously published results, but have statistical uncertainties that are $40 \%-50 \%$ smaller.

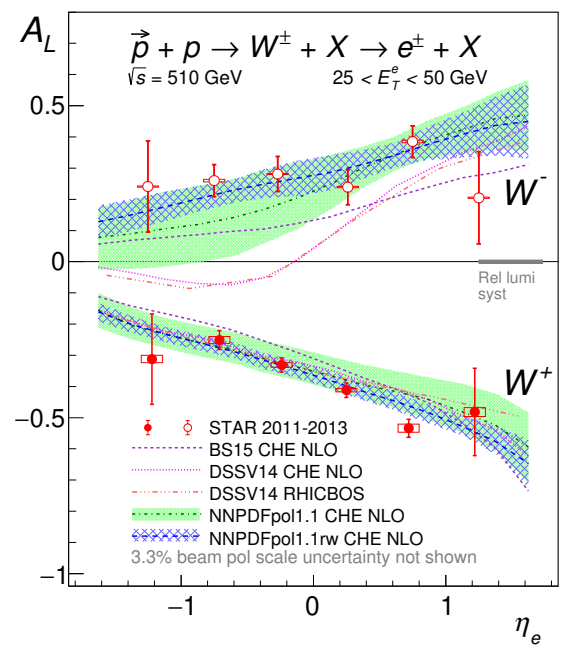

Figure 9. Longitudinal single-spin asymmetries, $A_{L}$, for $W^{ \pm}$production as a function of the positron or electron pseudorapidity, $\eta_{e}$, for the combined STAR 2011+2012 and 2013 data (points) in comparison to theory expectations (curves and bands).

The results are compared with expectations based on the DSSV14 [30], NNPDFpol1.1 [30] and BS15 [31] PDFs. The NNPDFpol1.1 analysis, unlike DSSV14 and BS15, includes the STAR $2011+2012 W^{ \pm}$data [32], which reduces in particular the uncertainties for $W^{-}$expectations at negative $\eta$. The data confirm the existence of a sizable, positive $\Delta \bar{u}$ in the range $0.05<x<0.25$ [32] and the existence of a flavor asymmetry in the polarized quark sea.

\section{Summary}

The recent results from the STAR experiment at RHIC has been overviewed. A comprehensive set of flow measurements for different colliding nuclei has been presented. The detailed comparisons of the measurements show the sensitivity of $v_{n}$ to the magnitude of the initialstate eccentricity, system size, and final-state interactions in the expanding matter. The $\Lambda$ polarization along the beam directions with a quadrupole structure has been observed for the first time and needs futher theoretical input. The values of coalescence parameter, $B_{2}$, for deuterons decrease as collision energy increases and seem to reach a minimum at about $\sqrt{s_{N N}}=20-40 \mathrm{GeV}$, indicating a change in the equation of state. $B_{2}$ values for antideuterons have been found to be less than those for deuterons at collision energies below $62.4 \mathrm{GeV}$. New STAR measurements of longitudinal single-spin asymmetry for $W^{ \pm}$produced in polarized proton-proton collisions at $\sqrt{s}=510 \mathrm{GeV}$ have been shown. The $A_{L}$ data for $W^{+}$ and $W^{-}$, combined with previously published STAR results, show a significant preference for $\Delta \bar{u}\left(x, Q^{2}\right)>\Delta \bar{d}\left(x, Q^{2}\right)$ in the fractional momentum range $0.05<x<0.25$ at a scale of $Q^{2}=10(\mathrm{GeV} / \mathrm{c})^{2}$. 


\section{Acknowledgements}

The reported study was partially supported the Ministry of Science and Higher Education of the Russian Federation, grant No 3.3380.2017/4.6, and by the National Research Nuclear University MEPhI in the framework of the Russian Academic Excellence Project (contract No. 02.a03.21.0005, 27.08.2013). The part of the work was performed using resources of NRNU MEPhI high-perfomance computing center.

\section{References}

[1] S. Voloshin, Y. Zhang, Z. Phys. C 70, 665 (1996)

[2] D. Teaney, L. Yan, Phys. Rev. C 83, 064904 (2011)

[3] M. Luzum, J.Y. Ollitrault, Phys. Rev. Lett. 106, 102301 (2011)

[4] J. Adam et al., Phys. Rev. Lett. 122, 102301 (2019)

[5] Z.T. Liang, X.N. Wang, Phys. Rev. Lett. 94, 102301 (2005)

[6] S. Voloshin (2004), 041008

[7] L. Adamczyk et al., Nature 548, 62 (2017)

[8] L. Adamczyk et al., Phys. Rev. C 98, 014910 (2018)

[9] I. Karpenko, F. Becattini, Eur. Phys. J. C 77, 213 (2017)

[10] H. Li, L.G. Pang, Q. Wang, X.L. Xia, Phys. Rev. C 96, 054908 (2017)

[11] J. Adam et al., Phys. Rev. Lett. 123, 132301 (2019)

[12] J. Adam et al., Phys. Rev. C 99, 064905 (2019)

[13] M.J. Bennett et al., Phys. Rev. C 58, 1155 (1998)

[14] T.A. Armstrong et al., Phys. Rev. C 61, 064908 (2000)

[15] T.A. Armstrong et al., Phys. Rev. Lett. 85, 2685 (2000)

[16] G. Ambrosini et al., Phys. Lett. B 417, 202 (1998)

[17] I.G. Bearden et al., Eur. Phys. J. C 23, 237 (2002)

[18] I.G. Bearden et al., Phys. Rev. C 94, 044906 (2016)

[19] C. Adler et al., Phys. Rev. Lett. 87, 262301 (2001)

[20] S.S. Adler et al., Phys. Rev. Lett. 94, 122302 (2005)

[21] M. Lisa et al., Phys. Rev. Lett. 84, 2798 (2000)

[22] L. Ahle et al., Phys. Rev. C 66, 054906 (2002)

[23] L. Adamczyk et al., Phys. Rev. C 92, 014904 (2015)

[24] K. Aamodt et al., Phys. Lett. B 696, 328 (2011)

[25] M.M. Aggarwal et al., Phys. Rev. C 83, 064905 (2011)

[26] J. Adam et al., Phys. Lett. B 797, 134917 (2019)

[27] J. Adam et al., J. High Energy Phys. 07, 051 (2015)

[28] A. Andronic, P. Braun-Munzinger, K. Redlich, J. Stachel, Nature 561, 321 (2018)

[29] J. Adam et al., Phys. Rev. D 99, 051102(R) (2019)

[30] D. de Florian, R. Sassot, M. Stratmann, W. Vogelsang, Phys. Rev. Lett. 113, 012001 (2014)

[31] C. Bourrely, J. Soffer, Nucl. Phys. A 941, 307 (2015)

[32] L. Adamczyk et al., Phys. Rev. Lett. 113, 072301 (2014) 